\title{
DOENÇA DE ALZHEIMER E DEMANDAS DE CUIDADOS: o que os enfermeiros sabem?
}

\author{
Silvana POLT RON IERE ${ }^{a}$, F átima Helena CECCHET T $0^{b}$, E miliane N ogueira de SOUZAc
}

\section{RESUMO}

0 objetivo deste estudo foi desvelar o conhecimento de enfermeiros de unidades de internação clínica acerca da D oença de Alzheimer (DA) e da demanda de cuidados de pacientes e familiares. Estudo descritivo-exploratório, com abordagem qual itativa. Os dados foram obtidos através de entrevista semi-estrutur ada. A satur ação dos dados ocor reu com 11 enfer meiros de dois hospitais privados. D a análise de conteúdo, emergiram seis categorias: caracterização da DA, fatores de risco, for mas de diagnóstico, complicações, medicações para tratamento, cuidados com doente e família. O bservou-se que os enfermeiros possuem um conhecimento limitado sobre a DA, focando as ações de cuidado na alteração clínica que motivou a internação hospitalar. Reconhecem sinais e sintomas, mas se mostram como figuras coadjuvantes na assistência, quando deveriam posicionar-se de for ma mais autônoma frente ao cuidado e à atenção à família.

D escritores: D oença de A Izheimer. Cuidados de enfermagem. Conhecimento.

\section{RESUMEN}

E I objetivo de este estudio fue revelar el conocimiento de enfermeros de unidades de internación clínica sobre la E nfermedad 0 $\mathrm{M}$ al de Alzheimer (E A) y las exigencias del cuidado de los pacientes y sus familias. E studio descriptivo exploratorio, con abordaje cualitativo. L os datos fueron obtenidos a través de entrevista semiestructurada. $L$ a saturación de datos se realizó con once enfermeros de dos hospitales privados. D el análisis de contenido, emergieron seis categorías: caracterización de la E A, factores deriesgo, formas dediagnóstico, complicaciones, medicación para tratamiento, cuidados con el enfermo y la familia. Se observó que los enfermeros poseen un conocimiento parcial sobre la E A, enfocando las acciones de cuidado en la alteración clínica quecausóla internación hospitalaria. Reconocen señales y síntomas, pero se muestran figuras coadyuvantes en la asistencia, cuando deberían posicionarse de forma más autónoma frente al cuidado y a la atención a la familia.

Descriptores: E nfermedad de Alzheimer. Atención de enfermería. Conocimiento. T ítulo: $\mathrm{M}$ al deAlzhei mer y demandas de cuidados: ¿Cuánto saben los enfermeros?

\section{ABST RACT}

Theaim of this study is to show what nurses from hospitalization units know about A lzheimer's disease (AD) and thedemands of carefor patients and their families. T his is a descriptive and explorator $y$ study with a qualitativeapproach. The data were collected through semistructured interviews. D ata saturation occurred after interviewing eleven nurses in tw o privatehospitals. Six categories emerged from the content analysis: AD characterization, risk factors, ways to diagnose, complications, treatment medication, patient and family care. It was obser ved that nurses have partial knowledge regarding AD, focusing their care actions only on the clinical change that caused admission. They recognize signs and symptoms but only show themselves as supporting assistants, instead of acting more autonomously in dealing with care needs and family support.

Descriptors: Alzheimer disease N ursing care Knowledge.

T itle: Alzheimer's disease and care needs: what do nurses know?

\footnotetext{
a Coordenadora da Associação Brasileira de A Izheimer, Enfermeira da Clínica Lar do I doso Bela Vista, Caxias do Sul, Rio Grande do Sul, Brasil.

${ }^{b}$ M estre em M edicina T ropical, Professora A ssistente da Faculdade Fátima, Caxias do Sul, Rio G rande do Sul, Brasil.

c M estre em Ciências da Saúde: Cardiologia, Professora A ssistente da U niversidade F ederal de Ciências da Saúde de Porto A legre (U F CSPA), Rio Grande do Sul, Brasil.
} 


\section{INT RODUÇÃO}

A Doença de Alzheimer corresponde, atualmente, à forma mais comum de demência, sendo a grande causa de comprometimento cognitivo e comportamental no envelhecimento. Estudos realizados em 2010 apontam que existem cerca de 35 milhões de casos no mundo, e sua prevalência vem aumentando de forma significativa ${ }^{(1)}$. Com base na expectativa de vida que aumenta em países em desenvolvimento, estima-se que, no mundo, em 2050, $22 \%$ da população seja composta por idosos. No Brasil, conforme estimativas para 2020, a expectativa de vida ultrapassará os setenta e cinco anos, chegando a $15 \%$ da população. N esse contexto, o Brasil será o sexto país no mundo com pessoas idosas, existindo uma expectativa de aumento de doenças crônico-degenerativas, dentre elas o Alzheimer ${ }^{(2-4)}$.

Dentre as 10 primeiras causas de óbitos nos Estados U nidos, a Doença de Alzheimer ocupa a 6 a posição no ranking, sendo a única que não tem como ser prevenida e tampouco curada ${ }^{(5)}$. A lém disso, a taxa de mortalidade das doenças que ocupam as primeiras posições, como as cardio e cerebrovasculares tem diminuído, enquanto que a taxa de óbito por Alzheimer tem aumentado(5). E mbora a Doença de Alzheimer seja uma doença progressiva e incurável, muito já se avançou em benefício e meIhoria da qualidade de vida dos portadores e cuidadores, com medicações que melhoram a cognição e diminuem as alterações comportamentais durante seu uso, além da criação de bons instrumentos de avaliação e de critérios diagnósticos mais claros.

Contudo, existe uma grande parcela de profissionais da área da saúde e cuidadores sem esclarecimentos norteadores sobre tal patologia, enfrentando, nas diversas fases da doença, a dúvida do que fazer, bem como do tipo de apoio que necessitam para enfrentar a doença em todo o seu longo curso ${ }^{(2,3)}$. Em se tratando de doença neurológica crônico-degenerativa, traz consigo dúvidas em relação ao manejo do doente, afetando aspectos de ordem pessoal, emocional, financeiro e social do paciente e seus familiares.

Assim, justifica-se este estudo que tem por objetivo desvelar o conhecimento de enfermeiros de unidades de internação clínica acerca da doença de A Izheimer e da demanda de cuidados de pacientes e familiares.

\section{MÉTODOS}

Trata-se de um estudo descritivo-exploratório, qualitativo, desenvolvido em duas instituições hospitalares, situadas no mesmo município da região nordeste do estado do Rio Grande do Sul, em abril de 2009. Para caracterizá-las, optou-se por renomeá-las: Instituição A conta com 90 leitos; Instituição B possui 144 leitos.

A população em estudo constitui-se de enfermeiros alocados em unidades de inter nação clíni$\mathrm{ca}$, num total de 22 profissionais nas referidas instituições hospitalares. Os critérios de inclusão foram: estar atuando a mais de 12 meses em um dos três turnos de trabalho (manhã, tarde ou noite), já ter realizado ações de cuidado aos pacientes portadores da doença de Alzheimer e seus familiares durante internação hospitalar, e concordância com o T ermo de Consentimento Livre e E sclarecido. A amostra fora definida por saturação dos dados, a partir da percepção e do domínio teórico do pesquisador de que idéias novas fossem acrescentadas, a ponto de oportunizar nova codificação. Para tal constatação, à medida que os participantes eram entrevistados, ocorria a transcrição das falas e a préanálise. Os dados foram obtidos através de entrevista $^{(6)}$ semi-estruturada, orientada por tópicos acerca da temática, elaborados pelos pesquisadores.

A entrevista foi realizada durante o turno e no local de trabalho dos enfermeiros, em horário previamente combinado entre uma das pesquisadoras e o entrevistado, através de ligação telefônica. As questões norteadoras dividiram-se em dois eixos temáticos: 0 conhecimento acerca da Doença de A Izheimer e os aspectos relacionados ao cuidado de enfermagem, com o portador da doença e sua família. As entrevistas foram registradas por meio de gravação em fita K-7. Os dados foram analisados através da anál ise de conteúdo ${ }^{(7)}$. E nvolveu as etapas de transcrição das falas, leitura flutuante, buscando saber qual a interpretação do entrevistado sobre a doença de Alzheimer e suas demandas de cuidado, a codificação (momento em que os dados brutos são transformados de forma organizada e agregados em unidades que permitam a descrição de características pertinentes ao conteúdo) e, por fim, a categorização (classes que reúnem um grupo de elementos em razão de características comuns) $)^{(7)}$.

Este estudo foi aprovado pelo Comitê de Ética da Instituição de origem, sob o número 027/ 08. 
Os participantes foram identificados com $\mathrm{S}_{1}, \mathrm{~S}_{2}$ e assim por diante, garantindo 0 anonimato dos mesmos.

\section{RESULT ADOS E DISCUSSÃO}

Foram entrevistados onze enfer meiros, sendo seis da instituição $A$ e cinco da instituição $B$. Do total de enfermeiros convidados a participar do estudo, três recusaram-se, dois estavam trabaIhando há menos de um ano, e um não havia cuidado de paciente com Alzheimer em sua trajetória de trabalho. A maioria dos enfermeiros é do sexo feminino (nove), sendo que sete deles tinham até cinco anos de formados. Seis enfermeiros responderam estar cursando ou já ter cursado pós-graduação, contudo, nenhum deles em áreas específicas, como geriatria ou gerontologia. Cinco enfermeiros não possuíam nenhuma especialização. A maioria era trabal hador do turno da tarde (sete).

As informações obtidas nas entrevistas semiestruturadas estão ag rupadas de acordo com o eixo temático, em seis categorias.

\section{Caracterização da doença de Alzheimer}

U ma das doenças que mais tem desafiado a medicina nos últimos anos é a Doença de Alzheimer. Sendo assim, ter um paciente internado em uma unidade clínica não é tarefa fácil para o enfermeiro, pois o saber sobre esta doença também se torna frágil, quando não estamos em uma área específica de assistência a pacientes neurológicos. 0 desabafo desse enfermeiro, no início da entrevista, demonstra essa inquietação:

[ ...] acredito ser uma doença bem progressiva, complicada. Q uando a gente tem paciente, nem sabe como tratar (S6).

A D oença de Alzheimer é uma doença neurológica, degenerativa, lenta e progressiva, deteriorando a memória breve, que costuma manifestar-se após os 50 anos $^{(8)}$. 0 paciente que por ela é atingido, apresenta uma crescente dificuldade em memorizar, decidir, agir e alimentar-se, até atingir 0 estado vegetativo. Os enfermeiros a seguir expressam-se de acordo com as idéias da autora:

[ ...] doença neurológica que, com o avançar da idade, vai aparecendo e piorando [ ...] (S4).
[ ...] é uma doença que atinge o sistema nervoso central e ocasiona perda de memória, o paciente fica confuso (S5).

Alguns autores relatam que a D oença de Alzheimer tem sido considerada, nos últimos anos, como uma doença heterogênea nos seus aspectos etiológicos e neuropatológicos, sendo a mais comum dentre o grupo das demências, tendo como principal sinal a perda de memória, sendo confundida com o processo do envelhecimento natural(8), compreendida pelos enfermeiros, através de suas falas:

[ ...] a Doença de Alzheimer começa com a deterioração da memória breve, é um processo evolutivo, degenerativo e sem retorno, não tem melhorar (S1).

[ ...] é uma doença degenerativa e traz complicações para o paciente se não for acompanhado (S2).

Cabe mencionar que, além da perda de memória, poderá haver a dificuldade de completar as atividades domésticas, confusão de tempo e espaço, alterações na fala durante a conver sação, diminuição da capacidade de julgamento e tomada de decisão, mudanças de humor e personalidade, bem como alterações na real ização rotineira de atividades diárias relacionadas ao autocuidado, lazer e trabalho.

No decorrer das entrevistas, percebeu-se uma confusão entre a D oença de A Izheimer e a doença de Parkinson, como pode ser observada nas falas a seguir.

Característica dessa doença? tremores, perda de força em membros inferiores e esquecimento [ ...] (S4).

Perda de memória, tremores [ ...] (S5).

É possível atribuir esta confusão com sinais típicos da doença de Parkinson, pelo fato de ser mais incidente em pessoas idosas. Como sintomas básicos da doença de Parkinson, citam-se os tremores em repouso, rigidez, encurvatura para a frente e perda de força motor $a^{\left({ }^{(9)}\right.}$. Da mesma forma que 0 A lzheimer, o Parkinson é degenerativo, porém esta doença pode ser estabilizada com fármacos específicos, não levando o paciente à perda de memória(9). 


\section{Fatores de risco para desenvolver Alzheimer}

M uito já se avançou em relação a essa implacável doença, mas pouco se sabe. A comunidade científica que estuda doenças degenerativas concorda apenas que 0 início desta patologia se dá por aspectos multifatoriais. Os fatores de risco conhecidos são: idade, história familiar positiva e síndrome de $D$ own ${ }^{(8)}$, sendo este um aspecto aparentemente desconhecido por dois dos entrevistados, como se percebe nos discursos abaixo:

Ah não sei [ ...] (S9).

N ão faço idéia [ ...] (S8).

Pensar em Alzheimer é pensar em trabalhar com a mente, ou pensar no que deixou de ser trabalhado com esta mente doente. Seguem as falas que vão ao encontro dessa inferência:

[ ...] A coisa que a gente mais ouve falar éa falta de exercício cerebral, precisa manter atividades cer ebral, precisa-se ler [ ...] (S1).

A não atividade, a falta de leitura. A prevenção éler livros, não só assistir T V, mas faz er com que o cér ebro faça uma prática um poucochinho mais aprimorada. As costureiras, por exemplo, eu não vejo ter a D oença deAlzheimer [...] (S6).

0 nível educacional é uma possível causa ambiental, mas os estudos que apontam essa causa são ainda demasiadamente controversos, faltando evidências ${ }^{(8)}$.

Outro fator de risco trazido pelos enfermeiros entrevistados é o fator her editário, como mostra a transcrição:

\section{[ ...] A genética também éresponsável (S3).}

N ão se sabe a causa. P ode ser genético [ ...] (S11).

A tríade idade, história familiar e genética constituem os fatores de risco mais importantes. 0 fato de ter um membro na família em 10 grau torna os demais propensos à incidência de Alzheimer. Em consonância, as mutações genéticas de três cromossomos (1,14 e 21) podem ser as responsáveis pelo início precoce da doença, embora represente $5 \%$ de todos os casos de Alzheimer entre trinta e cinquenta anos(8). Outro gene da A po- lipoproteína E (APOE), identificado no cromossomo 19, possui diferentes formas. Enquanto uma de suas formas parece evitar o desenvolvimento da doença, no caso do gene da APOE 4, a presença deste significa uma maior suscetibilidade, representando $65 \%$ de todos os pacientes de A lzheimer estudados. Contudo, os mesmos autores trazem que o fato de uma pessoa desenvolver a doença ligada à causa genética, não quer dizer que a prole terá(8).

Entende-se que a D oença de Alzheimer é tida como uma doença do envelhecer, fator de risco para o desenvolvimento. $M$ as não são raros os casos pré-senis em pessoas a partir de trinta anos, como o relato de Dr. Alois na descoberta da doença ${ }^{(10)}$.

A idade é, indiscutivelmente, o fator de risco mais importante para o desenvolvimento de demência e da D oença de Alzheimer, podendo ser constatada a partir de sessenta anos de idade. As falas a seguir evidenciam a idade como fator de risco:

[...] eu acho que éa idade, o paciente fica velho e dá nisso (S7).

\section{[ ...] acredito que com a idade também (S3).}

$\mathrm{N}$ a fala do S7, percebe-se a ideia de que 0 envelhecer carrega consigo as doenças neurológicas. No entanto, é importante destacar que a D oença de Alzheimer e o envelhecimento não são sinônimos, pois alterações cognitivas leves também podem ser encontradas no envelhecimento normal(8).

\section{Formas de diagnóstico da Doença de Alzheimer}

Considerando-se que o diagnóstico de Alzheimer é de presunção clínica, a percepção da família e de outros profissionais da área da saúde são fundamentais para que o médico possa estabelecer 0 diagnóstico. U ma história clínica adequada, a confirmação por parte dos familiares e a avaliação do estado mental pode conferir até $90 \%$ de precisão para o diagnóstico.

D entre os diversos exames que são utilizados para contribuir no diagnóstico de Alzheimer, estão exames laboratoriais, hemograma, creatinina, sódio e potássio, cálcio, dosagem de vitamina B12, sorologia para sífilis, função hepática, hormônio estimulante da Tireóide (T SH). Exames como 
tomografia computadorizada, ressonância magnética e a avaliação neuro-psicológica surgem como opcionais ${ }^{(8)}$. Sendo assim, a radiografia não está inclusa como um método de investigação, porém este recurso apareceu nas falas dos enfermeiros como opção diagnóstica:

\section{[ ...] Diagnóstico? A través de radiografia (S4).}

A avaliação médica inclui uma descrição detal hada do histórico do paciente, para que se possa iniciar um processo de investigação. I nformações sobre personalidade prévia, nível educacional, os hobbies e os históricos ocupacional e social podem servir não somente como auxílio importante para 0 diagnóstico ${ }^{(8)}$. Bem compreendido pelo enfermeiro S4, conforme descrito abaixo:

[ ...] acho quepelos sinais esintomas quea pessoa apre senta, que ela chega no profissional, ou familiar vai junto com o paciente, e diz que está acontecendo o esquecimento (S2).

As decisões devem ser pautadas não somente pela definição da extensão das aval iações e da confiabilidade dos métodos diagnósticos, mas também pela análise da relação custo-benefício e pelos riscos existentes ${ }^{(8)}$. É fundamental que os médicos estejam familiarizados com os significados dos resultados obtidos para o diagnóstico.

\section{Complicações da D oença de Alzheimer}

Os portadores de D oença de A Izheimer apresentam grande risco de pneumonias aspirativas e, em um estágio avançado, tornam-se acamados. Podem apresentar disfagia, que evolui para o uso de sondas enterais, úlceras de pressão, infecções pulmonares, embolias e outras patologias relacionadas à dependência(11).

E $m$ relação às patologias mais frequentes, os entrevistados sinalizaram que a pneumonia por aspiração é a mais comum, como corroboram os depoimentos abaixo:

[ ...] Q uando os pacientes já estão mais acamados, e desenvolvem uma outra patologia, como uma pneumonia, acabam morrendo por causa disso (S5).

[ ...] fica muito tempo acamado, então ele passa a ter problemas pulmonares, como a pneumonia por aspiração, causando até a morte(S8).
Outro evento citado pelos entrevistados como agravantes da doença, foram as fraturas. Sabese que as quedas em idosos podem ser bem limitadoras e até fatais. U m estudo que avaliou quedas nessa população mostrou dados alarmantes: $78 \%$ dos idosos que se acidentaram apresentavam diagnóstico de demências ${ }^{(12)}$. N este estudo, somente um dos entrevistados mencionou a queda como uma das causas de complicações:

[ ...] Principal causa de óbitos acredito ser a queda (S6).

Em virtude do declínio do equilíbrio e da instabilidade postural, associados ao uso de medicamentos para controle das alterações comportamentais, a queda é uma das causas de hospitalização, para investigação e tratamento de fraturas.

D evido à suscetibilidade do paciente idoso, sondagens de alívio ou de demora abrem portas para que patógenos oportunistas tomem conta do sítio de inserção. Dentre as respostas, percebeuse que os enfermeiros relacionam as infecções urinárias ocorridas em ambiente hospitalar como uma das complicações em pacientes com Doença de A Izheimer. Seguem as falas:

D os pacientes que a gente recebe, percebe se agravamento e óbi to por infecções de vias urinárias, é um tal de tira e bota de sonda eisso dá infecção [ ...] (S10).

[ ...] infecção urinária que a gente vê aqui [ ...] (S4).

E m estágio avançado da Doença, o autocontrole das eliminações fisiológicas pode estar prejudicado. N esse sentido, a avaliação da capacidade funcional pela enfermagem e equipe multidisciplinar torna-se essencial para o planejamento do cuidado. A partir de então, reconhecendo-se as limitações do paciente e as possibilidades de per manência hospitalar, pode-se implementar estratégias de cuidado que, além de atender às necessidades de cada paciente, também procuram evitar as complicações decorrentes de procedimentos invasivos.

\section{T ratamento da D oença de Alzheimer com medicamentos}

A pesar de não curar ou reverter a doença, determinados medicamentos auxiliam no bem-estar, minimizando problemas, como agitações, insônia e depressão ${ }^{(8)}$. Contudo, parte dos enfermeiros res- 
pondeu que havia medicamentos, porém desconhecia seus nomes ou princípios ativos, como pode ser percebido nos relatos a seguir:

E u sei que existem, mas não sei te dizer o nome, eles tentam retardar o processo de evolução da doença [...] (S1).

\section{[ ...] existe, mas não sei nome [ ...] (S3).}

Os medicamentos mais frequentemente prescritos pelos profissionais médicos são: Donepezil, Rivastigmina, $G$ alantamina, os quais são bem tolerados nas fases inicial e intermediária. Outro fármaco aliado ao controle da Doença de Alzheimer é a M emantina, que é utilizada nas fases intermediária e final, e auxilia a manter as funções de comunicação e atividade de vida diária( ${ }^{(8)}$. D entre os entrevistados, um enfermeiro soube citar o nome de medicamentos utilizados:

[ ...] Tem sim, eu me recordo, o E xelon e o A lois. Sei que tem outros também (S11).

$M$ ais importante que saber o nome comercial do medicamento, ésaber que eles existem. T ais medicamentos são utilizados para controle de sinais e sintomas cognitivos e comportamentais. U ma vez que o paciente esteja inter nado em ambiente hospitalar, torna-se fundamental assegurar a continuidade da administração de medicamentos de uso contínuo no domicílio.

\section{Cuidado com o doente e a família}

Entre as respostas mais encontradas dentre as dificuldades de manejo com pacientes portadores de Doença de Alzheimer internados nos hospitais pesquisados, estão as dificuldades com os familiares, conforme se nota nas falas:

[ ...] a maior dificuldadeélidar com os familiares, eles são o maior transtorno [ ...] (S1).

[ ...] aceitação da família, a família não aceita que o paciente está naquela situação [ ...] (S3).

$N$ as fal as anteriores, percebe-se a dificul dade de atuar junto aos familiares, caracterizando-os como um fator inconveniente no cenário de cuidado. No contexto da D oença de A Izheimer, a família deve ser vista como um importante agente de cui- dado. N esse sentido, a assistência de enfermagem pode ser co-planejada com o(s) familiar(es) cuidador(es).

0 cuidador-familiar do portador da Doença de Alzheimer sofre alterações no seu cotidiano e merece apoio e valorização por parte dos profissionais da saúde, na tentativa de reduzir a vulnerabilidade a que ambos está sendo imputada ${ }^{(13,14)}$. Entende-se, assim, que cabe ao enfermeiro criar estratégias de acolhimento e suporte aos familiares para lidar com a inter nação e as al ter ações decorrentes da D oença, levando-se em consideração a necessidade de mudanças na dinâmica familiar.

Alguns entrevistados constataram a fragilidade do conhecimento da categoria profissional em relação à doença e trouxeram esse fato como um dos aspectos dificultadores da atuação junto à família e pacientes.

A busca por estratégias para minimizar a sobrecarga e manejar a situação de cuidado, pode agregar o conhecimento e a experiência da enfermagem como uma importante contribuição(15,16). Seguem as falas:

[ ...] a maior dificuldade é o conhecimento dos enfermeiros que é pouco, a falta de informação também (S11).

[ ...] a equipe tem que estar ciente e saber lidar, entender a doença pra poder lidar com o paciente. E u vejo por aqui, a gente não tem conheci mento e sem conhecer bem a patologia, não sabemos como lidar com o paciente efamília (S9).

Quando os enfer meiros foram questionados sobre seu papel frente ao familiar de um portador da D oença de A Izheimer, a maioria dos entrevistados teve a oportunidade de refletir sobre suas funções quando tem sob seus cuidados pacientes e familiares.

0 enfermeiro é que teria que ensinar para a família o cuidado com o paciente, pra isso é necessário estudar, e orientar a família depois de se orientar [ ...] (S2).

As funções de orientar e educar surgiram como as principais formas de desempenhar seu papel junto aos familiares. E mbora concor dem que existam falhas, tais funções foram as mais apontadas pelos enfermeiros, como mostram as respostas a seguir: 
[ ...] precisa educar bem essa família, dar orientações, passar conhecimento para ter um cuidado melhor em casa ... mas acontece, falha educativa por que a gente é muito mais do fazer [ .... (S1).

[ ...] tem que orientar bastante a família, não entendem que eles têm perda de memória e ficam irritados, porque tem quefalar várias vezes a mesma coisa, então entra o trabal ho do enfermeiro de educar (S5).

E mbora educar e orientar sejam realmente importantes, quando se trata de uma doença em que o paciente tem dificuldades em agir em seu próprio beneficio, tais ações voltam-se também para a família ou cuidadores. 0 conhecimento, habilidade de manejo, atitudes autênticas, empatia, paciência, tolerância, entre outras características, devem constituir o perfil do profissional que vai atuar nos ramos da gerontogeriatria ${ }^{(17)}$. A capacidade de superar dificuldades e preconceitos, desenvolvendo habilidades especiais para lidar com o paciente portador Alzheimer e sua família se tor na fundamental. Estas qualidades são difíceis de serem conquistadas, frente ao cenário apontado pela fala do profissional que segue:

[ ...] se a gente tivesse tempo pra dispensar a família, a confiança seria conquistada, mas sempre se está com pressa (S6).

A fase avançada ou terminal da Doença de A lzheimer é descrita como fase das perdas significativas, diminui-se vocabulário, apetite e peso, e aparecem descontrole urinário e fecal, dependência progressiva do cuidador ${ }^{(18)}$. G eralmente é nesse estágio que os enfermeiros assistenciais têm contato com os pacientes, pois ficam mais suscetíveis a problemas que necessitem de internação hospitalar. Com isso, levantou-se o questionamento sobre um plano de cuidados específicos para essa fase.

A lguns enfermeiros demonstraram não conhecer a classificação de fases ou estágios que 0 portador de D oença de Alzheimer cursa ao longo da evolução clínica da patologia, mencionando itens como atividades de estímulo cerebral, pintura, música, cuidados com excesso de deambulação, entre outros, a serem incluídos em um plano de cuidados para pacientes em fase terminal, como evidenciam as falas:

[ ...] atividades de pintura dá pra fazer, conversar bastante com esse paciente, caminhar com ele [ ...] (S6).
A tividades de leitura, cuidar com fugas de casa, que esses idosos podem se perder [ ...] (S8).

T endo em vista que o paciente que se encontra na fase avançada de A Izheimer permanece mais tempo no leito, a mudança de decúbito associada a demais cuidados, torna-se fundamental para prevenção de lesões de pele. Os enfermeiros concordam sobre a existência desse item num plano de cuidados:

[ ...] movimentação com frequência desse paciente, para que não surjam escaras (S1).

Orientar quanto à mudança de decúbito de duas em duas horas é primordial [ ...] (S3).

Tanto no ambiente hospitalar como no domicílio, a frequente mobilização no leito previne complicações que poderão acarretar maior carga de trabalho, além de mais preocupação ao cuidador.

Conhecendo a magnitude da Doença de Alzheimer (DA), dois enfermeiros mostraram-se engajados em cuidar do cuidador, uma vez que o suporte ao doente situa-se no âmbito de cuidados paliativos. Seguem as falas dos enfermeiros sob a ótica do cuidado humanizado:

[ ...] é impor tante que não se perca o vinculo e estabe leça uma relação de confor to para o familiar, preparar a família para a perda, principalmente nesse momento por quea dor vai ser grande [...] (S11).

[ ...] conhecimento da causa, saber e conhecer para depois trabal har, o enfermeiro precisa ter um ol har para o psicológico e elaborar um plano junto com a família (S8).

I mportante mencionar que, mesmo em cenários de cuidados voltados para o manejo de alterações clínicas ou cirúrgicas em portadores de D oença de Alzheimer, é oportuna a atenção aos familiares. São el es que poderão evitar novos episódios, tais como aqueles que motivaram a internação. A lém disso, torna-se importante a valorização do cuidador e do papel da família.

\section{CONSIDERAÇÕES FINAIS}

Observou-se que os enfermeiros das unidades de internação possuem um conhecimento li- 
mitado sobre a temática em questão. Reconhecem, na maioria das vezes, sinais e sintomas característicos, possíveis complicações, mas desconhecem aspectos relacionados ao manejo da doença. A demanda de cuidados está voltada para as patologias agudas que originam a inter nação, em detrimento dos aspectos relacionados à evolução da doença e às necessidades de atenção às limitações cognitivas e comportamentais do paciente, bem como às necessidade de apoio e orientações à família acometida pela Doença de Alzheimer em um de seus membros. Os profissionais em questão mostram-se como figuras coadjuvantes na assistência, quando deveriam posicionar-se de forma mais autônoma frente ao cuidado e a atenção à família de um portador de Alzheimer, oportunizando momentos de educação e orientação.

A partir dos achados desta pesquisa, somamse subsídios para a qualificação do cuidado oferecido aos enfermeiros, por meio de educação continuada em serviço, discussão de casos, incentivos à participação em eventos sobre a temática e busca por especialização profissional, para que a assistência de enfermagem seja otimizada, criando espaços de promoção da saúde para pacientes e famílias que convivem com essa doença crônico-degener ativa.

\section{REFERÊNCIAS}

1 Alzheimer's Disease International. W orld A Izheimer report 2010 [ Internet]. London; 2010 [ cited 2011 Feb 10] . Available from: http:/ / www.alz.org/ documents/ national/World_Alzheimer_Report_ 2010.pdf.

2 M inistério da Saúde (BR), Secretaria de Atenção a Saúde, D epartamento de A tenção Básica. E nvelhecimento e saúde da pessoa idosa [ Internet]. Brasília (D F ); 2007 [ citado 2011 fev 14] . (Cadernos de A tenção Básica; 19). D isponível em: http:/ / bvsms.saude. gov.br/ bvs/ publicacoes/ abcad19.pdf.

3 M oreira M M . D eterminantes demog ráficos do enveIhecimento brasileiro [ Inter net] . Campinas: A ssociação Brasileira de E studos Populacionais; 2000 [ citado 2011 fev 14]. Disponível em: http:/ / www.abep. nepo.unicamp.br/docs/ anais/ pdf/2000/ Todos/ idot5_1.pdf.

4 W orld Health Organization. G lobal age-friendly cities: a guide [ Inter net] . G eneva; 2007 [ cited 2011 Feb 14]. Available from: http:// who.int/ ageing/ publications/G lobal_age_friendly_cities_Guide_ English.pdf.
5 A Izheimer's D isease International. T he prevalence of dementia worldwide [ I nternet] . L ondon; 2008 [ cited 2011 M ar 03] . Available from: http:/ / www.alz.co.uk/ adi/ pdf/ preval ence.pdf.

6 Cervo AL, Bervian PA. M etodologia científica. São Paulo: M cG raw-Hill; 2002.

7 Bardin L. Análise de conteúdo. Lisboa: E dições 70; 2002.

8 Lokvig J, Becker JD. A lzheimer de A a Z. São Paulo: Verus; 2005.

9 Pinheiro J. Distúrbio do movimento: doença de Parkinson e não Parkinson. In: F reitas EV, Ly P, Cançado FAX, G orzoni M L. T ratado de geriatria e gerontologia. 2 a ed. Rio de Janeiro: Guanabara Koogan; 2006. p. 355-60.

10 M achado JC. O utras causas de demências: demências "potencialmente reversíveis". In: F reitas E V, Ly P, Cançado FAX, G orzoni M L. T ratado de geriatria e gerontologia. $2^{2}$ ed. Rio de Janeiro: G uanabara Koogan; 2006. p. 309-19.

11 Luzardo AR, G orini MIPC, Silva APSS. Características de idosos com doença de A Izheimer e seus cuidadores: uma série de casos em um serviço de neurogeriatria. T exto Contexto Enferm. 2006;15(4):58794.

12 Carvalho A M , Coutinho EF S. Demência como fator de risco para fraturas graves em idosos. Rev Saúde Pública. 2002;36(4):448-54.

13 Batistella M, Celich KLS. Ser cuidador familiar do portador de doença de A Izheimer : vivências e sentimentos desvelados. Cogitare Enferm. 2007;12(2): 143-9.

14 A rruda M C, Alvarez A M , G onçalves L HT. O familiar cuidador de portador de doença de Alzheimer participante deum grupo de ajuda mútua. Ciênc Cuid Saúde. 2008;7(3):339-45.

15 G ómez G ómez M M . Estar ahí, al cuidado de un paciente con demencia. Invest E duc E nferm. 2007;25 (2):60-71.

16 Santana RF, A Imeida KS, Savoldi NAM . Indicativos de aplicabilidade das orientações de enfermagem no cotidiano de cuidadores de portadores de Alzheimer. Rev Esc Enferm USP. 2009;43(2):45964. 
17 Zimerman GI. Velhice: aspectos biopsicossociais. Porto alegre: Artmed; 2000.
18 Canineu PR, Caovilla VP. Vocênão está sozinho. São Paulo: A braz; 2002.
E ndereço da autora / Dirección del autor / Author's address:

E miliane N ogueira de Souza

Rua Sarmento L eite, 245, ap. 411, Centro

90050-170, Porto Alegre, RS

E-mail:emilianes@ufcspa.edu.br
Recebido em: 31/ 05/2010

A provado em: 01/ 06/ 2011 